\title{
A Bibliometric Analysis of Publications on COVID-19 and Older Adults
}

\author{
Rabia Bag Soytas \\ Division of Geriatrics, Department of Internal Medicine, Cerrahpaşa Faculty of Medicine, Istanbul University-Cerrahpaşa, Istanbul, Turkey
}

Corresponding Author:

Rabia Bag Soytas, MD

Division of Geriatrics, Department of

Internal Medicine, Cerrahpaşa Faculty

of Medicine, Istanbul University-

Cerrahpasa, Kocamustafapasa Cad.

No:34/E Fatih, Istanbul, Turkey

E-mail: drrabiabag@gmail.com

ORCID:

https://orcid.org/0000-0002-9620-7596

Received: June 11, 2021

Revised: July 5, 2021

Accepted: July 6, 2021
Background: Bibliometric analysis is important for guiding future research priorities. We evaluated the most relevant scientific research on coronavirus disease 2019 (COVID-19) and older adults, analyzed current hot topics, and identified the 50 most cited publications. Methods: Articles published between December 1, 2019 and March 17, 2021 were identified using the search terms "COVID-19" or "Novel Coronavirus" or "SARS-CoV-2" or "2019-nCoV" and "geriatrics" or "older adults" or "elderly" appearing in the title, abstract, keywords, or keywords plus. Original research articles, reviews, editorial materials, and letters were included. Information on articles year, journal, title, author, country, affiliation, keywords, document type, and counts of citations was collected. VOSviewer was used to analyze keywords. Results: A total of 784 publications were included. The most common keywords were "COVID-19" and "older adults," which were strongly related to "social isolation," "dementia," "mortality," and "loneliness." The most active (40.8\%) and most cited $(1,578)$ country was the United States. The Journal of the American Geriatric Society had the largest number of publications (22.7\%) and citations (947). The most researched $(84.0 \%)$ and most cited areas were geriatrics-gerontology $(2,882)$. The median number of citations for the most cited 50 articles was 46.8. Conclusion: The results of the bibliometric analysis provided information about the quality and research areas of published studies on COVID-19 and older adults. Social and psychological support, nutrition, vaccines, and telemedicine may be hot research topics for the future.

Key Words: Bibliometrics, COVID-19, Geriatrics, Older adults, Web of Science

\section{INTRODUCTION}

The pneumonia outbreak that started in Wuhan, China in December 2019 and became a global pandemic was named coronavirus disease 2019 (COVID-19) and was caused by the newly identified severe acute respiratory syndrome coronavirus 2 (SARS-CoV-2). ${ }^{1)}$ As of March 5, 2021, over 110 million cases and over 2.5 million deaths have been reported worldwide. ${ }^{2)}$

Many diseases cause greater morbidity and mortality in patients aged 65 years and older owing to the frailty, comorbidities, and age-related physiological changes in this population. ${ }^{3)}$ Older adults are at serious risk for COVID-19 and also have higher mortality rates owing to the disease. ${ }^{4-6)}$ Furthermore, older adults have experienced social and psychological effects, apart from the disease, during the pandemic., ${ }^{7,8)}$

Bibliometry is a statistical method that determines which topics are trending by quantitatively analyzing articles in a particular field.9 The results of bibliometric analyses may guide future studies by determining the quality and main research areas of existing publications in specific fields. Bibliometric analysis also enables researchers to easily obtain information about subjects of interest from among numerous and increasing number of published articles. The Web of Science (WoS) is an online database comprising almost all relevant research articles and various bibliometric analysis tools. Bibliometric analysis studies have been reported on various fields of medicine. ${ }^{10,11)}$

The pandemic has not yet been controlled, and older adults are the most affected population. Therefore, bibliometric analysis of 
COVID-19 and older adults is important to identify related subjects that require further research by determining which areas have been researched thus far and which have been most cited. However, to date, there has been no bibliometric analysis of COVID-19 in older adults. Thus, to guide future research priorities, the present study evaluated the most relevant scientific research on COVID-19 and older adults, analyzed current hot topics, and identified the 50 most cited publications.

\section{MATERIALS AND METHODS}

The data used in this bibliometric citation analysis were obtained from the Thomson Reuters WoS Core Collection database (Philadelphia, PA, USA). As the WoS database provides bibliometric analysis, it has been used in most previous bibliometric analysis studies. Therefore, we also used this database. We scanned the global literature on geriatric COVID-19 published between December 1, 2019 and March 17, 2021 for articles containing the terms "COVID-19" or "Novel Coronavirus" or "SARS-CoV-2" or "2019-nCoV" and "geriatrics" or "older adults" or "elderly" in the title, abstract, keywords, or keywords plus (additional relevant but overlooked keywords not listed by an article's author or publisher but identified by Thomson Reuters). The corresponding author then reviewed the titles of all articles. We also read the abstracts of articles whose titles were not clear and excluded articles not related to either COVID-19 or older adults. Missing article information was obtained by linking to other search platforms such as PubMed. The examination of the titles, abstracts, keywords, or keywords plus of studies on older adults and geriatrics showed that some studies included patients aged 60 years, while others included patients aged 65 years. We included studies that performed subgroup analyses of older adults. The keywords were the most common terms in all research fields and were selected using a combination of the research results from multiple search queries in the WoS Core Collection. We recorded article information such as the year of publication, journal, title, author, country, affiliation, keywords, document type, and citation counts, all of which were exported to CSV format. We defined authorship as primary and co-authorship. We also recorded authors' countries and affiliations for each article. If more than one author of an article had the same country or affiliation, only one author was recorded. The number of citations was determined using WoS database analyses to identify the top 50 cited articles. From these results, we also recorded the journals in which the top 50 articles were published, article type, country of the corresponding author, publication date, and number of citations. VOSviewer (version 1.6.16, https: //www.vosviewer.com) is a software tool for constructing and visualizing bibliometric networks that we used to analyze keyword frequency to clarify the research trends on COVID-19 in older adults.

\section{Statistical Analysis}

We applied descriptive statistical methods using IBM SPSS Statistics for Windows, version 20.0 (IBM Corp., Armonk, NY, USA). All data are reported as numbers, percentages, and bar charts in the tables and figures.

\section{RESULTS}

We identified a total of 2,728 articles. After excluding articles that were not relevant to "COVID-19 and older adults," we analyzed 784 publications on COVID-19 and older adults indexed in the WoS database between December 1, 2019 and March 17, 2021, which included 350 (49.0\%) original research articles, $58(8.1 \%)$ review articles, 123 (17.3\%) editorial materials, and 182 (25.5\%) letters (Fig. 1). Among them, 63 (7.6\%) papers were published in 2021 (through March 17), and the other 650 articles were published in 2020. No papers on COVID-19 and older adults were published in 2019. Almost all the publications (710, 99.6\%) were written in English (two German and one French).

More than 1,200 institutions in 70 countries or regions have reported the results of COVID-19 research in older adults. The leading countries in the literature analyzed were the United States (291 reports, $40.8 \%$ ), China ( 84 reports, $11.8 \%$ ), Canada (62 reports, $8.7 \%$ ), England (59 reports, $8.3 \%$ ), and Italy (58 reports, $8.1 \%$ ). The most cited countries were the United States (1,578), China (815), England (433), Canada (432), and Italy (340) (Table 1). The most active organizations were Harvard University (35,

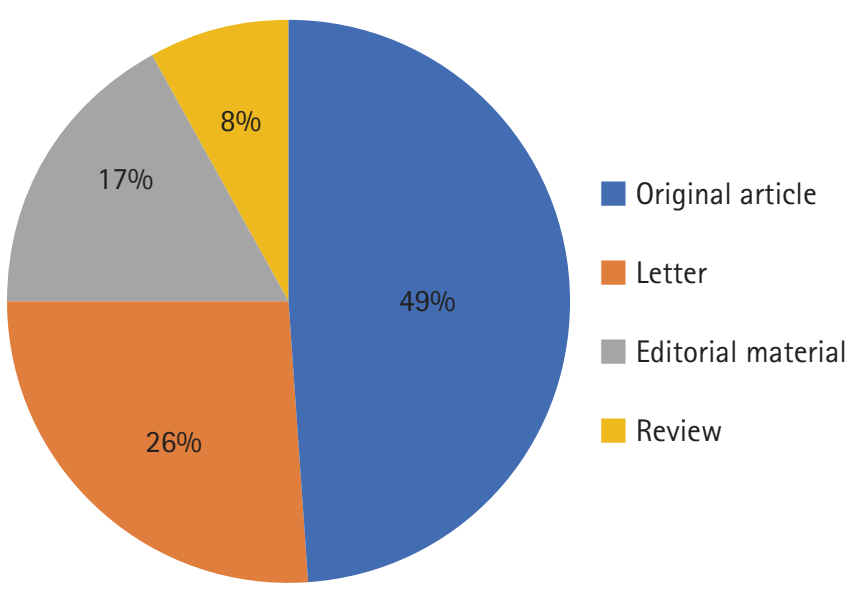

Fig. 1. Document types of publications on coronavirus disease 2019 (COVID-19) and older adults. 
4.9\%), US Veterans Health Administration (34, 4.8\%), and University of California System (26, 4.6\%), while the most cited organizations were Harvard University (245), University of London (237), and University of California (202) (Table 1).

The Journal of the American Geriatric Society had the highest number of publications $(178,22.7 \%)$, followed by the Journal of Gerontological Social Work (61, 7.8\%) and The American Journal of Geriatric Psychiatry (57, 7.3\%). The most cited journals were The Journal of the American Geriatric Society (947), American Journal of Geriatric Psychiatry (283), and Journal of Nutrition, Health and Aging (271) (Table 2).

The most highly researched areas were geriatrics-gerontology (599, 84.0\%), psychiatry $(143,20.0 \%)$, and social work (58, $8.1 \%)$. The most cited research areas were geriatrics-gerontology $(2,882)$, psychiatry (670), and nutrition dietetics (395) (Fig. 2). The most cited author was Joseph G. Ouslander from Florida Atlantic University/Charles E. Schmidt College of Medicine, with 10 articles and 159 citations (Table 2).

Keywords that were provided by the authors and that occurred more than five times in the WoS core database were enrolled in the final analysis. Among the 1,028 keywords, 49 met this threshold. The most common keywords were "COVID-19" (total link strength 231) and "older adults" (total link strength 102), which were strongly linked to "social isolation," "dementia," "mortality," and "loneliness" (Fig. 3).

In addition to the analysis of 784 publications on COVID-19 and older adults, the top 50 most cited publications are listed in Supplementary Table S1. These 50 articles had a median of 46.8 citations (range, 18-312 citations).

\section{DISCUSSION}

This is the first bibliometric study to summarize the most relevant evidence on COVID-19 in older adults. Evidence is quickly accumulating because this is a vulnerable population with worse outcomes. The COVID-19 pandemic has affected the entire world in a short time. While vaccination is becoming widespread, some countries still cannot obtain the vaccine. ${ }^{12)}$ Although the efficiency of existing vaccines is expected to be high, confirmatory data are lacking. ${ }^{13)}$ Moreover, as shown in this bibliometric analysis, few studies have focused on vaccination in older adults. It is unclear whether these findings will remain consistent by the time the pandemic ends. Thus, it is essential to support older adults and protect them from both COVID-19 and pandemic-related social and psy-

Table 1. The most active countries and organizations associated to publications on COVID-19 and older adults

\begin{tabular}{|c|c|c|}
\hline & Number of publications & Number of citations \\
\hline \multicolumn{3}{|l|}{ Top 10 countries } \\
\hline USA & $291(40.8)$ & 1,578 \\
\hline China & $84(11.8)$ & 815 \\
\hline Canada & $62(8.7)$ & 432 \\
\hline England & $59(8.3)$ & 433 \\
\hline Italy & $58(8.1)$ & 340 \\
\hline France & $38(5.3)$ & 173 \\
\hline Spain & $37(5.1)$ & 86 \\
\hline Japan & $27(3.8)$ & 98 \\
\hline Australia & $19(2.7)$ & 137 \\
\hline Netherlands & $17(2.4)$ & 93 \\
\hline \multicolumn{3}{|l|}{ Top 10 organizations } \\
\hline Harvard University & $35(4.9)$ & 245 \\
\hline US Veterans Health Administration & $34(4.8)$ & 103 \\
\hline University of California System & $26(4.6)$ & 202 \\
\hline University of London & $26(4.6)$ & 237 \\
\hline Geriatric Research Education Clinical Center & $24(3.4)$ & 102 \\
\hline Institut National de la Sante et de la Recherche Medicale & $22(3.1)$ & 49 \\
\hline University of Toronto & $20(2.8)$ & 196 \\
\hline Pennsylvania Commonwealth System of Higher Education & $14(2.0)$ & 145 \\
\hline State University System of Florida & $14(2.0)$ & 174 \\
\hline University of Michigan & $14(2.0)$ & 133 \\
\hline
\end{tabular}

Values are presented as number (\%).

COVID-19, coronavirus disease 2019. 
Table 2. The most active journals and authors of publications on COVID-19 and older adults

\begin{tabular}{|c|c|c|}
\hline & Number of publications & Count of citations \\
\hline \multicolumn{3}{|l|}{ Top 10 journals } \\
\hline Journal of the American Geriatrics Society & $178(22.7)$ & 947 \\
\hline Journal of Gerontological Social Work & $61(7.8)$ & 87 \\
\hline American Journal of Geriatric Psychiatry & $57(7.3)$ & 283 \\
\hline International Psychogeriatrics & $48(6.1)$ & 161 \\
\hline Aging-US & $43(5.5)$ & 174 \\
\hline Journal of Nutrition Health Aging & $43(5.5)$ & 271 \\
\hline Age and Ageing & $36(4.6)$ & 210 \\
\hline Frontiers in Psychiatry & $35(4.5)$ & 26 \\
\hline Journal of Aging Social Policy & $33(4.2)$ & 191 \\
\hline Geriatrics Gerontology International & $31(4.0)$ & 65 \\
\hline \multicolumn{3}{|l|}{ Top 10 authors } \\
\hline Ouslander, Joseph G. & 10 (4 first author) & 159 \\
\hline Annweiler, Cedric & 7 (1 first author) & 30 \\
\hline Arai, Hidenori & 7 (0 first author) & 48 \\
\hline Cesari, Matteo & 7 (1 first author) & 40 \\
\hline Rolland, Yves & 6 (2 first author) & 16 \\
\hline Lapid, Maria I. & 5 (2 first author) & 13 \\
\hline Morley, John E. & 5 (3 first author) & 99 \\
\hline Reynolds, Charles F. & 5 (3 first author) & 36 \\
\hline Rosen, Tony & 5 (3 first author) & 33 \\
\hline Sacco, Guillaume & 5 (2 first author) & 15 \\
\hline
\end{tabular}

Values are presented as number (\%).

COVID-19, coronavirus disease 2019.

chological problems. Knowledge of existing publications on older patients and COVID-19 may help researchers better understand issues that require further research.

The results of the current study showed that the United States had the highest number of publications on COVID-19 and older adults. This finding could be related to several factors such as its large population, its status as one of the countries most affected by the pandemic, its significant resources for medical research, and its well-developed and reliable data management systems. The Journal of the American Geriatric Society, one of the journals with a high impact factor, had the highest number of publications and citations on COVID-19 and older adults.

Most publications were in the fields of geriatrics-gerontology and psychiatry. Although there have been few studies on nutrition, it was the third most cited topic after geriatrics-gerontology and psychiatry. This result suggests that this topic is of interest, and further research is needed.

Among the top 50 cited publications of COVID-19 in older adults, the most cited articles were reviews. Seven articles were based on long-term care. Among these publications, Italy was the non-English-speaking and European country with the highest number of articles.
The results of the current study showed that "COVID-19" and "older adults," the two most prevalent keywords, were strongly linked to "social isolation," "dementia," "mortality," and "loneliness." These keywords were strongly linked likely because the disease mortality was the highest in older adults whose mental health is affected by restrictions and social isolation.

The most cited article was "Clinical features of COVID-19 in elderly patients: a comparison with young and middle-aged patients" published in The Journal of Infection. This article focused on factors associated with COVID-19 severity and mortality in older adults, such as the presence of malignancy, and also demonstrated higher COVID-19 mortality and severity in older adults compared to younger people. ${ }^{14)}$ Moreover, the 784 publications included in the present study mostly investigated factors affecting mortality in older adults and suggested the need for future research based on these factors (such as the presence of malignancy, symptoms of dyspnea, high neutrophil-to-lymphocyte ratio, $\mathrm{C}$ reactive protein level, lymphopenia, and basic biological mechanisms of aging). ${ }^{15-18)}$ The hotspots of future research on this subject may be prospective studies on reducing COVID-19 mortality in older adults.

The second most cited article, "Mental health services for older adults in China during the COVID-19 outbreak," published in The 


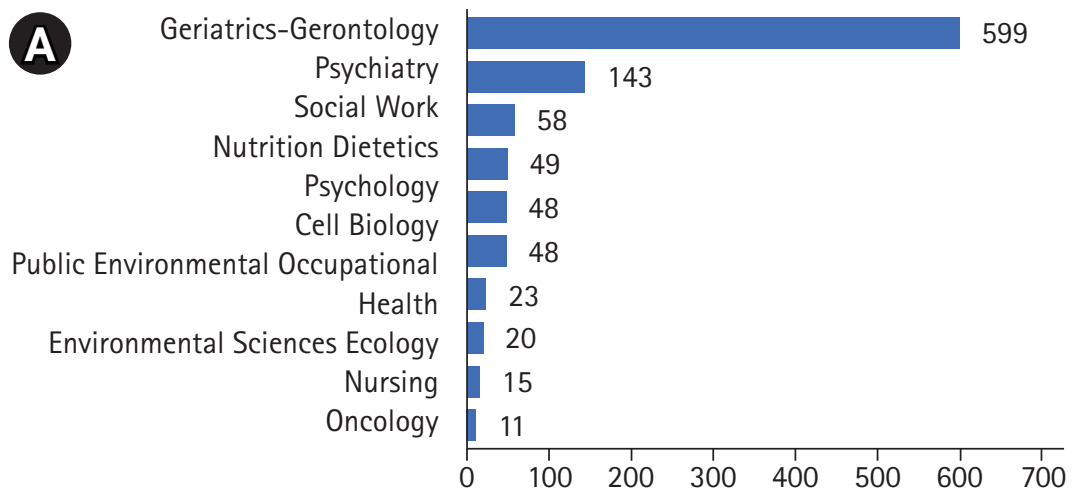

Number of publications

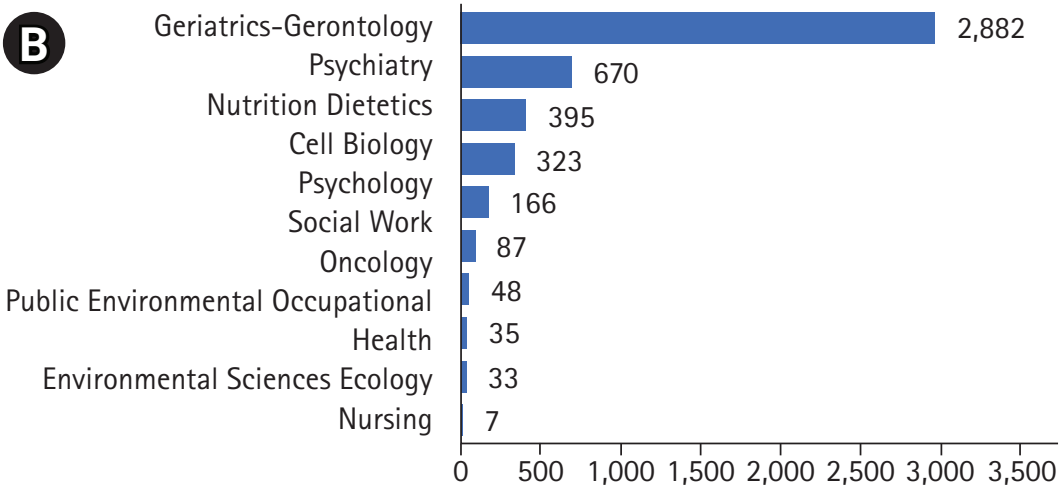

Number of publications
Fig. 2. Most active research areas related to coronavirus disease 2019 (COVID-19) and older adults. (A) Top 10 research areas with the highest numbers of publications on COVID-19 and older adults. (B) Top 10 research areas with the highest numbers of citations among publications on COVID-19 and older adults.
Lancet Psychiatry, emphasized the effects of the pandemic on mental health in older adults with increased depression. Along with increased depression, the study also reported decreased cognitive functions and consequently daily functionality. Therefore, there is a need for psychological services for older adults. ${ }^{19)}$ Retrospective studies reported increased suicide rates among older adults during the past SARS outbreak. ${ }^{20)}$ This situation highlights the importance of examining the impact of COVID-19 on mental health in older adults and finding solutions to prevent the pandemic from adversely affecting their mental health. Based on this identified need, The American Journal of Geriatric Psychiatry proposed to act as a forum for research on COVID-19 and mental health in older adults. $^{21)}$ One study, which was among the 50 most cited articles, reported risk factors that may increase suicide and proposed solutions for prevention in older adults during COVID-19. ${ }^{22)}$ Future studies may include the implementation and results of these proposed solutions to protect mental health in older adults.

Dementia was strongly linked to the most prevalent keywords. A study examining the effect of the COVID-19 pandemic on Alzheimer's dementia $(\mathrm{AD})$ reported that COVID-19 increases mortality and morbidity in patients with $\mathrm{AD}$ and may have affected $\mathrm{AD}$ diagnosis and clinical management, ${ }^{23)}$ emphasizing that the clinical presentation of COVID-19 may be atypical in dementia patients and demonstrating that identifying symptoms in these patients may be challenging owing to cognitive dysfunction, resulting in potential delays in diagnosis and hospitalization. ${ }^{24)}$ Thus, many studies have been conducted on dementia and COVID-19, and there is a need for additional studies that provide recommendations and practices to allow close follow-up of patients with dementia.

Loneliness has become an increasing problem for older adults in recent years. Several group interventions have been described to help alleviate loneliness in older adults. Among these, "Circle of Friends" is an evidence-based intervention that has proven to be effective and sustainable for the socialization of older adults and has been adapted to telehealth during the pandemic. ${ }^{25)}$ Additional studies are needed to develop telehealth and online group interventions to help lonely and isolated older adults to connect. Among the 50 most cited articles, some included loneliness in older adults owing to social isolation and the importance of supporting older adults on these issues. Among these, some studies also proposed solutions, including staying connected with relatives; ex- 


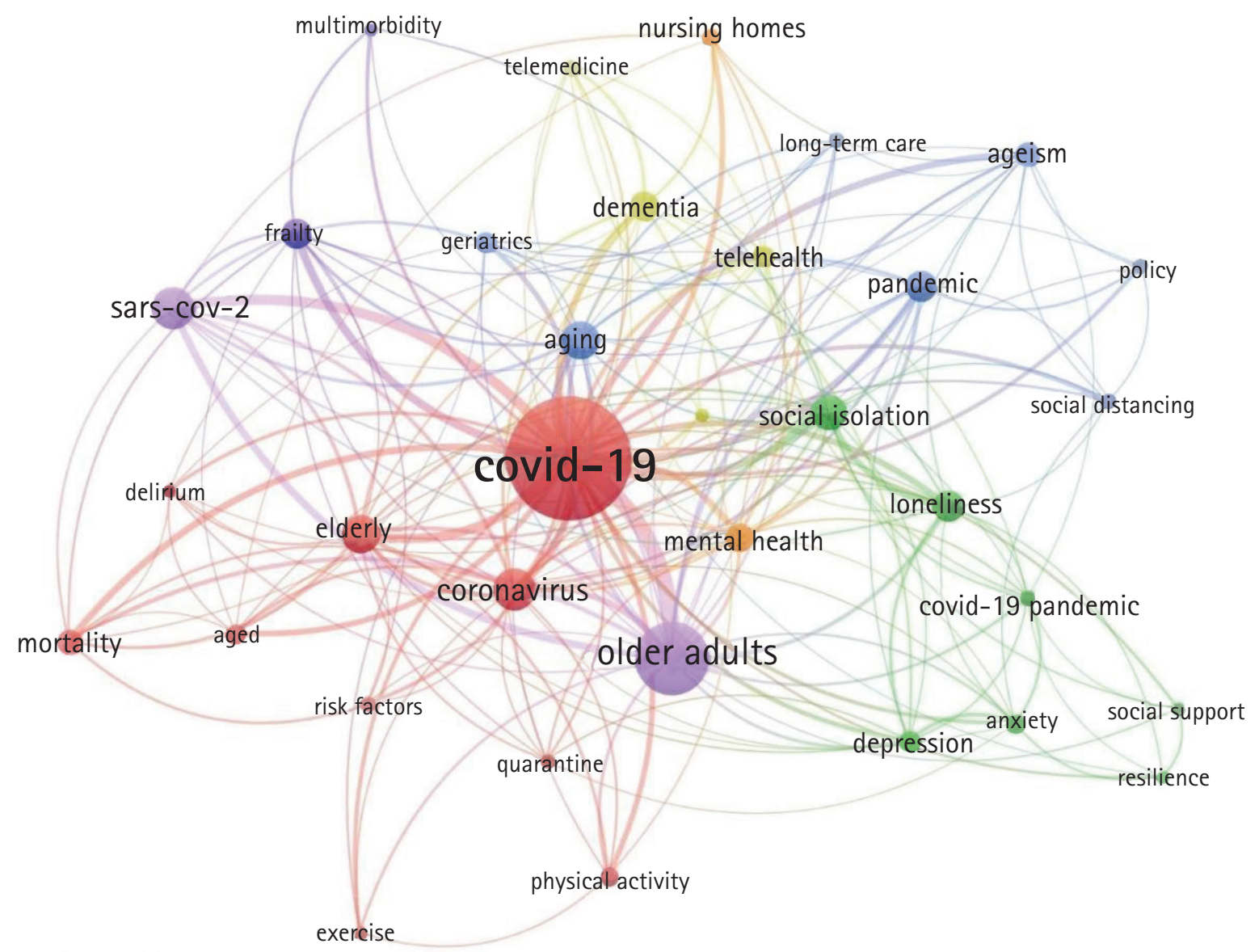

Fig. 3. Bibliometric analysis of the keywords in publications on coronavirus disease 2019 (COVID-19) and older adults.

ercising; practicing telehealth; and using digital resources, online tools, and social media. ${ }^{26,27)}$ The current data highlight the need to develop projects focusing on psychological services to support older adults during the pandemic. Furthermore, as technology advances, the importance of telemedicine has increased, which has been made obvious in the medical literature. It is essential to provide this service to older adults who cannot visit the hospital owing to the pandemic. As long as the pandemic continues, the need for research on telemedicine is likely to increase. Therefore, this may be an important future research topic.

The current study had strengths and limitations. The strengths of the study are that it provided quick access to the core of evidence around a topic and identified articles focused on older adults. One of the limitations was that it searched only one database (WoS Core Collection database); thus, it did not collect all available evidence. In addition, we did not analyze co-authorship and the relationships between publications and research groups. With changes in results over time and additional publications, future analyses could re-evaluate these findings at the end of the pandemic.
In conclusion, the results of the present bibliometric analysis revealed that most research has evaluated mortality, social isolation, dementia, and loneliness in COVID-19 and older adults. Most studies have been conducted to reveal these problems. Future hot research topics may include social and psychological support, vaccination, nutrition, and telemedicine, all of which could be the means to address the problems identified.

\section{SUPPLEMENTARY MATERIALS}

Supplementary materials can be found via https://doi.org/10.4235/ agmr.21.0060.

\section{ACKNOWLEDGMENTS}

\section{CONFLICT OF INTEREST}

The researcher claims no conflicts of interest.

\section{FUNDING}

None. 


\section{REFERENCES}

1. Wang C, Horby PW, Hayden FG, Gao GF. A novel coronavirus outbreak of global health concern. Lancet 2020;395:470-3.

2. World Health Organization. Coronavirus disease (COVID-19) Dashboard [Internet]. Geneva, Switzerland: World Health Organization; 2021 [cited 2021 Jul 15]. Available online: https:// covid19.who.int/.

3. Dent E, Morley JE, Cruz-Jentoft AJ, Woodhouse L, Rodriguez-Manas L, Fried LP, et al. Physical frailty: ICFSR International Clinical Practice Guidelines for Identification and Management. J Nutr Health Aging 2019;23:771-87.

4. Landi F, Barillaro C, Bellieni A, Brandi V, Carfi A, D'Angelo M, et al. The new challenge of geriatrics: saving frail older people from the SARS-COV-2 pandemic infection. J Nutr Health Aging 2020;24:466-70.

5. Shahid Z, Kalayanamitra R, McClafferty B, Kepko D, Ramgobin D, Patel R, et al. COVID-19 and older adults: what we know. J Am Geriatr Soc 2020;68:926-9.

6. Ng Cheong Chung KJ. The frailty and mortality relationship in patients with COVID-19. Eur Geriatr Med 2021;12:213-4.

7. Shrira A, Hoffman Y, Bodner E, Palgi Y. COVID-19-related loneliness and psychiatric symptoms among older adults: the buffering role of subjective age. Am J Geriatr Psychiatry 2020;28:12004.

8. Brooke J, Jackson D. Older people and COVID-19: isolation, risk and ageism. J Clin Nurs 2020;29:2044-6.

9. Ellegaard O, Wallin JA. The bibliometric analysis of scholarly production: how great is the impact? Scientometrics 2015;105: 1809-31.

10. Yu Y, Li Y, Zhang Z, Gu Z, Zhong H, Zha Q, et al. A bibliometric analysis using VOSviewer of publications on COVID-19. Ann Transl Med 2020;8:816.

11. Wang P, Tian D. Bibliometric analysis of global scientific research on COVID-19. J Biosaf Biosecur 2021;3:4-9.

12. Our World in Data. Statistics and Research: Coronavirus (COVID-19) vaccinations [Internet]. Oxfordshire, UK: Our World in Data; 2021 [cited 2021 Jul 15]. Available from: https:// ourworldindata.org/covid-vaccinations/.

13. Hodgson SH, Mansatta K, Mallett G, Harris V, Emary KR, Pollard AJ. What defines an efficacious COVID-19 vaccine? A review of the challenges assessing the clinical efficacy of vaccines against SARS-CoV-2. Lancet Infect Dis 2021;21:e26-e35.

14. Liu K, Chen Y, Lin R, Han K. Clinical features of COVID-19 in elderly patients: a comparison with young and middle-aged patients. J Infect 2020;80:e14-e18.

15. Sun H, Ning R, Tao Y, Yu C, Deng X, Zhao C, et al. Risk factors for mortality in 244 older adults with COVID-19 in Wuhan, China: a retrospective study.J Am Geriatr Soc 2020;68:E19-E23.

16. Leung C. Risk factors for predicting mortality in elderly patients with COVID-19: a review of clinical data in China. Mech Ageing Dev 2020;188:111255.

17. Promislow DE. A geroscience perspective on COVID-19 mortality.J Gerontol A Biol Sci Med Sci 2020;75:e30-e33.

18. Bag Soytas R, Unal D, Arman P, Suzan V, Emiroglu Gedik T, Can $\mathrm{G}$, et al. Factors affecting mortality in geriatric patients hospitalized with COVID-19. Turk J Med Sci 2021;51:454-63.

19. Yang Y, Li W, Zhang Q, Zhang L, Cheung T, Xiang YT. Mental health services for older adults in China during the COVID-19 outbreak. Lancet Psychiatry 2020;7:e19.

20. Yip PS, Cheung YT, Chau PH, Law YW. The impact of epidemic outbreak: the case of severe acute respiratory syndrome (SARS) and suicide among older adults in Hong Kong. Crisis 2010;31: 86-92.

21. Vahia IV, Blazer DG, Smith GS, Karp JF, Steffens DC, Forester BP, et al. COVID-19, mental health and aging: a need for new knowledge to bridge science and service. Am J Geriatr Psychiatry 2020;28:695-7.

22. Wand AP, Zhong BL, Chiu HF, Draper B, De Leo D. COVID-19: the implications for suicide in older adults. Int Psychogeriatr 2020;32:1225-30.

23. Brown EE, Kumar S, Rajji TK, Pollock BG, Mulsant BH. Anticipating and mitigating the impact of the COVID-19 pandemic on Alzheimer's disease and related dementias. Am J Geriatr Psychiatry 2020;28:712-21.

24. Bianchetti A, Rozzini R, Guerini F, Boffelli S, Ranieri P, Minelli $\mathrm{G}$, et al. Clinical presentation of COVID19 in dementia patients. J Nutr Health Aging 2020;24:560-2.

25. Zubatsky M, Berg-Weger M, Morley J. Using telehealth groups to combat loneliness in older adults through COVID-19. J Am Geriatr Soc 2020;68:1678-9.

26. Berg-Weger M, Morley JE. Editorial: Loneliness and social isolation in older adults during the COVID-19 pandemic: implications for gerontological social work. J Nutr Health Aging 2020; 24:456-8.

27. Hwang TJ, Rabheru K, Peisah C, Reichman W, Ikeda M. Loneliness and social isolation during the COVID-19 pandemic. Int Psychogeriatr 2020;32:1217-20. 